\title{
SPATIAL MODELS FOR ARCHITECTURAL HERITAGE IN URBAN DATABASE CONTEXT
}

\author{
E. Costamagna*, A. Spanò* \\ * Politecnico di Torino, DINSE, Architecture faculty Viale Mattioli 39 - 10125 - Torino \\ erik.costamagna@polito.it, antonia.spano@polito.it
}

KEY WORDS: spatial models, 3D GIS, CH documentation

\begin{abstract}
Despite the GIS (Geographic Information Systems/Geospatial Information Systems) have been provided with several applications to manage the two-dimensional geometric information and arrange the topological relations among different spatial primitives, most of these systems have limited capabilities to manage the three-dimensional space. Other tools, such as CAD systems, have already achieved a full capability of representing 3D data. Most of the researches in the field of GIS have underlined the necessity of a full 3D management capability which is not yet achieved by the available systems (Rahman, Pilouk 2008) (Zlatanova 2002). First of all to reach this goal is important to define the spatial data model, which is at the same time a geometric and topological model and so integrating these two aspects in relation to the database management efficiency and documentation purposes. The application field on which these model can be tested is the spatial data managing of Architectural Heritage documentation, to evaluate the pertinence of these spatial models to the requested scale for the needs of such a documentation. Most of the important aspects are the integration of metric data originated from different sources and the representation and management of multiscale data. The issues connected with the representation of objects at higher LOD than the ones defined by the CityGML will be taken into account. The aim of this paper is then to investigate which are the favorable application of a framework in order to integrate two different approaches: architectural heritage spatial documentation and urban scale spatial data management.
\end{abstract}

\section{INTRODUCTION: PRELIMINARY ISSUES IN CH SPATIAL DATA PROCESSING AND MANAGING}

The products of $\mathrm{CH}$ metric documentation usually come from different sources like detailed surveys, photogrammetric plotting and LiDAR scanning. The row data are processed and structured in order to provide an integrated 3D representation of architectural buildings and monumental complexes, the geometrical and thematic features of the output may be very different according to the documentation purposes.

These aims play a central role also in the organisation of these features in a hierarchy of different detail levels in order to perform specific queries and relate the complexes of buildings with the urban context. Usually the range of the scale of architectural representations is comprised between the 1:500 to 1:20 and sometimes over. The 1:500 scale is the closest to the urban scale and it's usually used to describe aggregates of buildings, like monumental complexes. Usually this kind of objects like the archaeological sites are compound by several buildings which may be interconnected or separated, each one of them with distinguishing marks and relations with the complex established during a certain life phase of the monumental building.

The typical scales of the architectural planning are the 1:200 and 1:100 which are used to describe the building in its exterior and interior aspect, usually through $2 \mathrm{D}$ sections or projections and $3 \mathrm{D}$ models. In the range of the architectural representation scales this medium level of detail is capable to render all the main building parts such as structural and decorative elements in their essential geometrical feature. The 1:50 and 1:20 scales are targeted to interior planning and for describing architectural or archaeological elements or other details. This high level is usually required to represent architectural details such as friezes or other decorative elements, but also the surface appearance of the objects in relation to the materials features or the deterioration state, usually with the support of ortho-projected or rectified photos.
The typologies of the output may be detailed 2D vector and raster drawings, usually with a graphical symbolization displaying the semantic value of the geometrical elements and/or a thematic classification of the objects in different layers, ortho-projected and rectified photos, 2.5D and 3D representations like TINs, texturized surfaces and solid models. During the different processing stages we are used to switch between this different kind of representations to comply the documentation purposes and the needs of the final users. Pointclouds and elevation models may be processed in order to extract 2D sections or detailed 3D textured models, but the same traditional $2 \mathrm{D}$ representations may also serve the purpose to build the 3D models. The choice between the different typologies of graphical output depends, of course, on the level of detail but also on the features of the surveyed object. For example buildings whose elements are well defined through simple geometrical primitives are efficiently build through the interpolation of 2D sections, whereas less regular surfaces, like for example architectural heritage or archaeological evidences are best rendered through surface models obtained through point-clouds interpolations.

The higher-scale and 3D representations are usually performed with $\mathrm{CAD}$ applications, targeted to this scale of representation, because of its good attitude to manage different typologies of $2 \mathrm{D}$ and $3 \mathrm{D}$ geometrical elements such as different kinds of curves (Bezier, splines), parametric solids, parametric surfaces and NURBS surfaces. CAD systems are also able to perform $3 \mathrm{D}$ realistic renderings with detailed definition of the surface appearance like materials mapping, lighting effects and so on. On the contrary GIS applications are focused on analysis and representations at smaller scales and are usually applied for urban and environmental analysis. The capability of 3D visualization and high-detailed outputs are usually limited and often the simple texture mapping of a facade may be an problem to overcome resorting to technical tricks like importing linked geometry whose material definitions are already build in CAD environment. GIS have many tools to perform the tasks usually required in this field, like for example georeferencing, data interpolations and several analysis on the thematic and 
geometrical features. On the other side CAD applications have limited or no capabilities to manages spatial and thematic data in a database structure, so one of the major problem, in the passage from GIS to CAD, is the loss of semantic informations associated with the geometrical features (Oosterom 2006).

The acquired experience in managing this kind of metric products let us to point out the need of integrated frameworks and analysis tools to manage all the aspects of $\mathrm{CH}$ metric documentation. This aim is assumed in order to overcome the loss of information in the passage between different kinds of application frameworks and perform efficiently analysis on the three-dimensional component of spatial data, which is essential in the architectural representation and may be useful for the purposes of $\mathrm{CH}$ documentation.
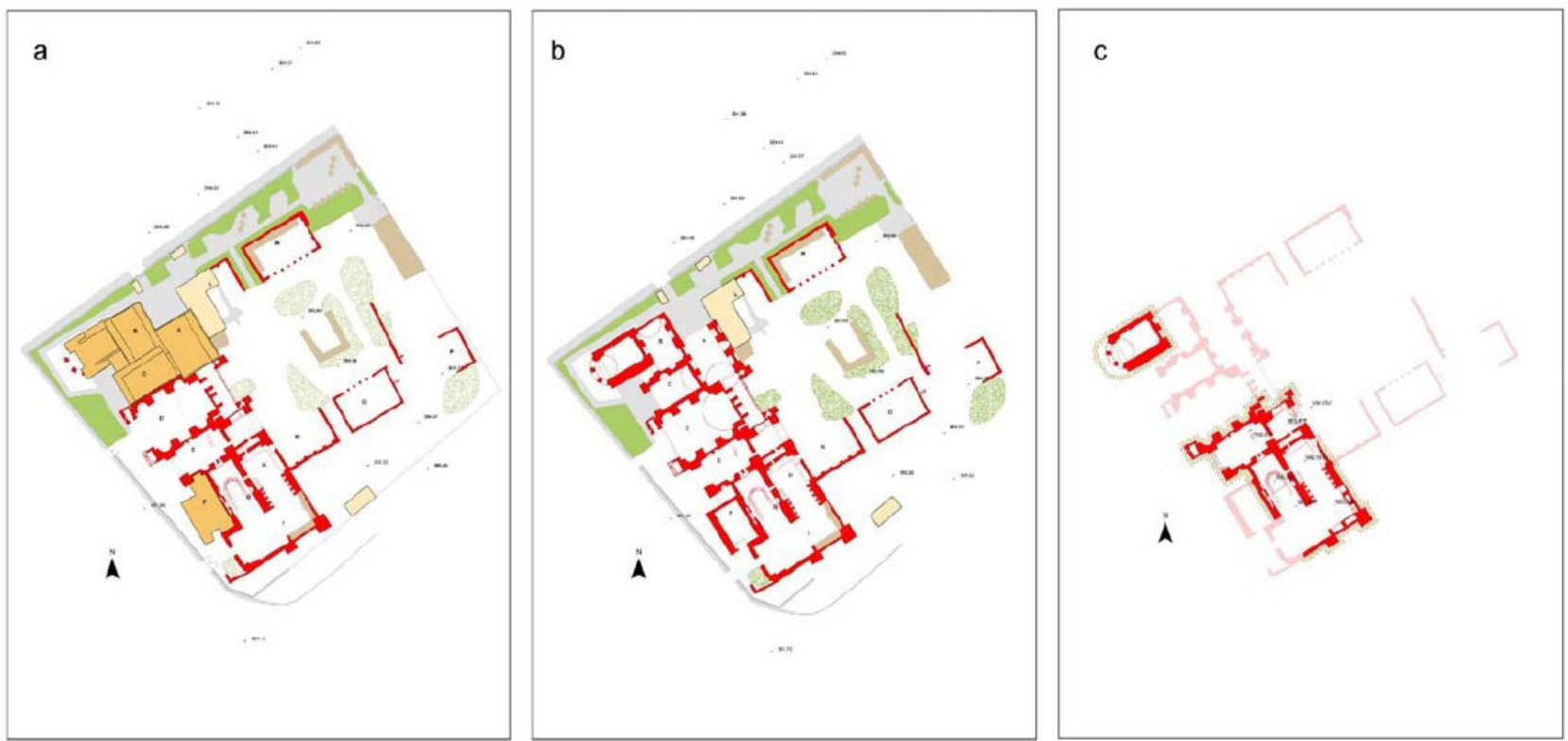

Figure 1: An archaeological map based on topographical database has been developed. Some spatial overlay based on third dimension connotation have been achieved: in the sequence picture each representation shows different planimetric section level. They describe roof level (a), ground level (b) and excations level (c) of the Big Bath in Hierapolis of Phrygia (Turkey).

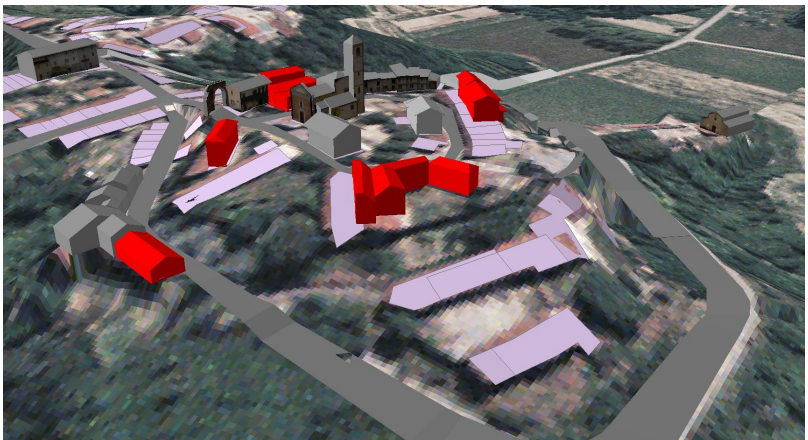

Figure 2: $3 D$ representation in GIS environment of a small rural village (Bagnasco AT) with texturized facades using linked geometry
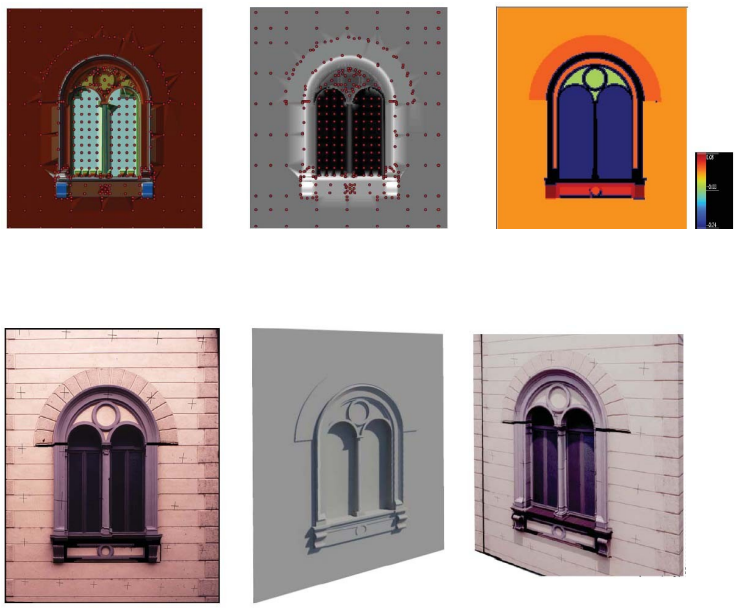

Figure 3: different level of details of documentation requirements demand different survey system. Continuous sufaces acquiring methods request suitable classification of elements strategies.In the picture sequence different processing phases (stereoplotting, dem processing, ortophoto and orthophoto $3 D$ processing for a double lancet window). elaboration Presti.G. 

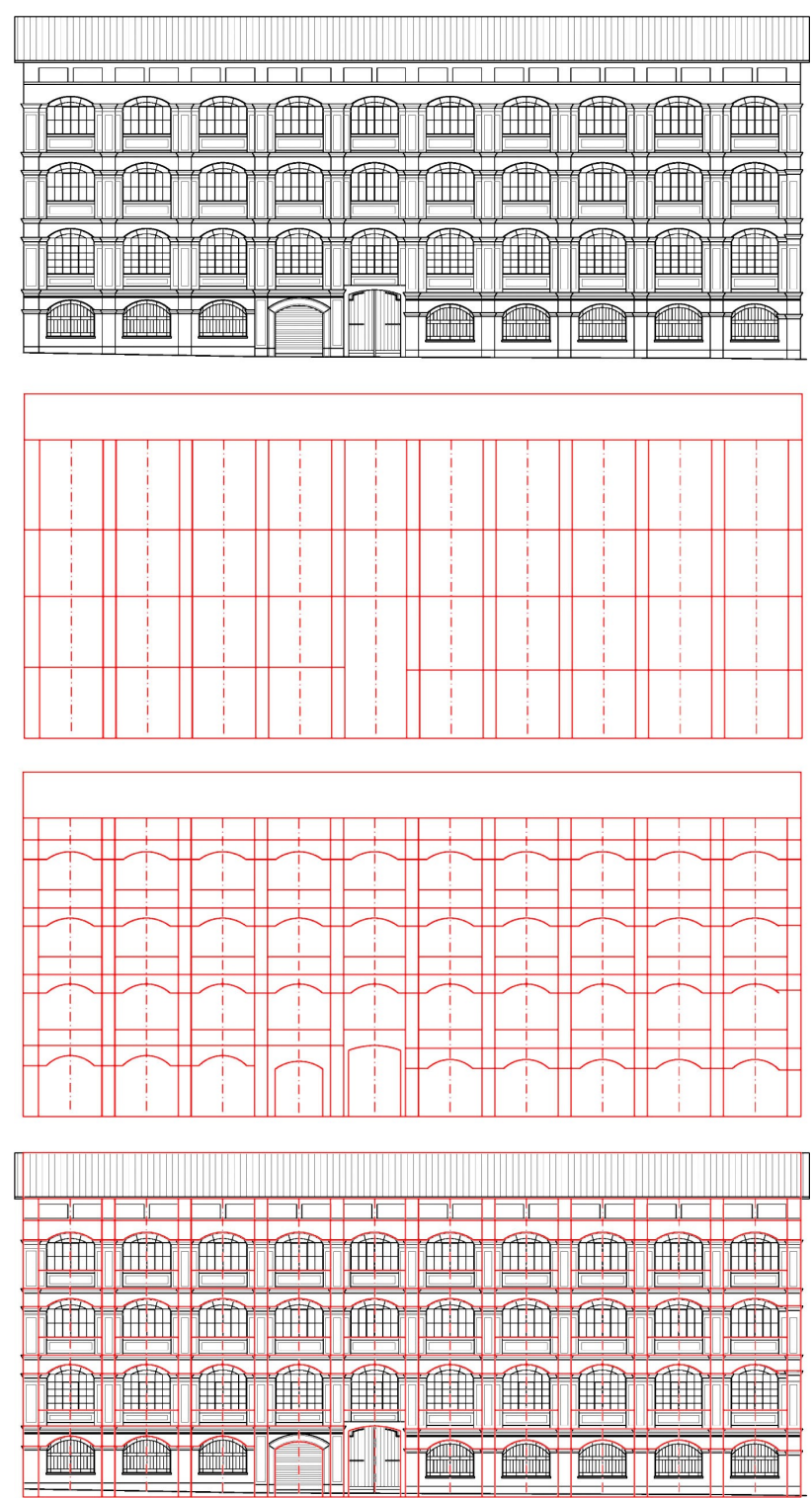

Figure 4:The pictures sequence shows the different $2 D$ drawings structuring according to different scale of representation for a building façade. An attemptable $3 D$ modeling of the same façade should adopt armonized kinds of schematizing.

\section{OVERVIEW OF 3D SPATIAL MODELS}

Considering the aspects of the management of 3D spatial data for $\mathrm{CH}$ documentation underlined in the introduction, a brief overview of spatial models can be made focusing on the capability of them to manage and represent high-detailed geometrical features and different kinds of data structures like the survey products we are used to manage.

Several spatial models have been proposed starting from the late '80s and each one shows aspects which make them more suitable for a specific field of application, from the terrain to the urban modelling. The selection of the appropriate model depends essentially on the typology of the geometric objects we're going to represent and on the complexity degree of the structure of spatial objects and theirs aggregations.

As underlined by Molenaar [MolenaAr 1998] and others one of the first classification of spatial models is based on the way the model represents the geometrical objects in the space: in the field-approach the real world objects are treated as a continuum in which the thematic classes of the spatial representations are linked directly to the geometric data and so the attributes are represented like a function of the position. This kind of models, in spite of the simple mathematical formulation, aren't capable to store explicitly the topological relationships. For this reason they're used mainly in the modelling of natural features where the geometrical objects doesn't have a value that can be described independently from the position in the space. This kind of features can be easily mapped through a direct association of the thematic attributes to the position in the space. An example of field-based model is the cell-tuple model proposed by Pigot (Pigot 1991).

On the contrary in the object-structured approach the space is considered as an empty container in which the objects are represented with their geometric and thematic aspects. This approach can be considered as a formalisation of the geometrical space in which objects are structured regardless of the dimensional and positional features. Each objects, stored independently with an unique identifier, is linked both with the thematic and geometric data. So, instead of a direct link between position and theme, in the object-structured approach the mapping of the thematic features is done through the object identifier. This identifier fulfil the purpose of storing the geometrical features independently from the position in the space clarifying the kind of geometry represented (lines, surface and solids for example are declared). It can also be used to store explicitly the topological relationship of the geometrical elements.

Other classifications can be made, for example, on the thematic representation of objects: in SVVMs (Single Valued Vector Map) like 3D FDS only one thematic class can be assigned to each geometrical object and so there is a 1:1 relationship between topological primitives and geometric elements of the same dimension, while in the MVVMs (Multi Valued Vector Map) several values for the thematic classes can be assigned. In other words in the MVVMs singularities of the same dimension are admitted, thus geometrical elements of the same dimension belonging to the same feature class cannot coexist in the same space.

Some preliminary remarks can be made on these spatial models regarding the geometrical and topological structure. Founding on the cell-graphs representation of the topological structure of geometrical elements different types of primitives are allowed in each model and consequently different spatial relationships can be stored in the model. The basis condition of this formalism is that in each geometrical representation the geometry can be described through a set of elementary geometrical objects, called simplices, which are «the simplest polyhedrons of each dimension» (Frank, Khun 1986).

Some examples of the object-structured approach are briefly exposed.

\subsection{The 3D FDS (3D Formal Data Structure)}

The 3D FDS (Formal Data Structure) (Molenaar 1990) is a geometrical formalism consisting in a set of definitions and rules for the spatial modelling: the topological primitives are 4: node, arc, face and edge and the geometrical simplest entities are also 4: point, line, surface and body. The edge primitive can be regarded as a 2D topological representation of an arc: in fact an arc can represent a 1D line or it can assumes the role of an edge between two adjacent faces (see diagram schema in Molenaar 1990). The 3D representation of a solid is obtained 
through the definition of the body primitive. There are limitations, for example arcs must be straight lines and the faces must be planar, otherwise faces can have arbitrary number of nodes and an edge can be formed by several arcs. The relationship between edge and face is explicitly stored (arc-face relationship) and consequently the orientation of a face is described through the direction of the edges. Also other relationships like node-on-face, arc-on-face, node-in-body, arc-in-body and face-in-body are explicitly stored. This representation is one of the most suitable for the spatial analysis due to the fact that, in this model, every geometry element can be defined through the relationships with the constitutive primitives of the lower dimension (i.e. a face is described by the composing arcs and an arc through the nodes). This structure allows also an explicit storage of topological relationship between adjacent elements (i.e. an arc belonging to 2 faces is stored only one time and it can be used to link two adjacent geometries).

\subsection{The TEtrahedral Network (TEN)}

The TEN model (Pilouk 1996) is based on the simplicial-complex model (Carlson 1987). According to the simplicial-complex model three simplest geometry are defined for each dimension: point ( 0 -simplex), line (1-simplex), triangle (2-simplex), tetrahedron (3-simplex); each of the primitives (excluding the 0 -simplex) can be used to model aggregations (complexes) of the same dimension. This simple framework allows a direct storage of topological relationships both of the constitutive parts of a 3D geometry and the adjacent objects. Two nodes define univocally a line, three lines a triangle and so a tetrahedron through triangles (i.e. in a $3 \mathrm{D}$ geometry in the triangle table are stored links to the tetrahedron, triangles and edges). This model belongs to the class of the full space decomposition and it has a $3 \mathrm{D}$ primitive which is the tetrahedron (Penninga 2008).

\subsection{The Simplified Spatial Model (SSM)}

The SSM (Simplified Spatial Model) (Zlatanova 2000) is a model focused on the query aspects and web accessing. It has some similarities with the 3D FDS model and it's called simplified due to the fact that arcs are omitted from the allowed primitives. As the 3D FDS model in the SSM the geometrical elements are 4: point, line, surface and body; otherwise the primitives are only 2 : node and face. The omission of the arc comes from a series of considerations: a line object or an edge can be represented easily through a series of nodes and the omission of the arc table lead to a remarkable reduction of data storage. Relationship between node-in-face and face-in-body are stored and the orientation of faces are described by means of the nodes sequence. In 3D representations the relationship between arc and faces may be not unique (unlike the 2D space case where it's used to link two adjacent faces) and so in this case the role of the edge primitive is not essential.

\subsection{The Urban Data Model (UDM)}

The UDM (Urban Data Model) (Coors 2002) is similar to the SSM model and contains some adding restrictions. As the in 3D FDS there are four geometrical elements: point, line, surface and body and as the SSM there are only 2 kind of topological primitives: node and face. The adjacency of two faces is therefore defined by the sharing of at least two nodes. In the relational implementation faces are decomposed into triangles, but while this represent an increase of storage data, every face table contains only three columns. The singularities node-in-face and arc-on-face are so resolved.

\subsection{Object-oriented models}

Consequently to the spread of the object-oriented programming languages the object modelling approach was applied to the data modelling in the DBMS (Data Base Management Systems). According to the Golden Rules of the Manifesto (Atkinsons 1990) the main differences between the object-oriented paradigm and the relational one, defined by Codd (Codd 1970), can be traced in relation to the aspects involving the spatial typology of data and the procedures executed on them. In the OODBMS (Object-Oriented Data Base Systems) data are organized in classes representing the domain of the objects which are considered as instances of a class. Classes group together objects with similar aspects such as the thematic classes of urban furnitures in a city model dataset. The inheritance is one of the main difference between the object-oriented approach and the relational: starting form a super classes several different level of sub classes can be defined and each one of them inherits the attributes from the parent class. This feature allows the modelling of complex aggregations of objects. An other aspect of this approach is the encapsulation which mean that the procedures to manage data are embedded in the data itself. That means the logical independence of data, which can be modified without changing the languages used to access to them. Consequently of the encapsulation and inheritance the same procedure on a object may determine a different behaviour from a class to the subclasses and it's called polymorphism. This means more freedom for the user to create new classes with their own specific procedures. OODBMS are also extensible i.e. new kind of classes and methods can be created without restructuring the whole DBMS and without the need to change the language used to manage data.

Based upon this principles some applications of 3D spatial models have been made in OODBMS. One of them is the 3D TIN developed by Rahman (Rahman 2000) (Rahman 2008) based upon the 3D FDS schema.

In this schema the more abstracted level of description of spatial data is represented by the TSpatial object class from which is derived the TGeometry class. All geometrical primitives are derived from the TGeometry class. Four primitives are defined: node, edge, polygon and solid. This classes are used to store the topological relationships like point-line, point-surface, line-surface and line-solid, while the lower classes (XYZContainer and ARContainer) are used as a repository from the upper classes to retrieve the geometry. The object-oriented schema is also used to model the process of the 3D TIN generation from a 3D point grid. Starting from the distance transformation class (TDT), whose instances are used by the 3D Voronoi class (TVor) to tessellate the space. The TVor class is used by the TTGeneration (TTinGen) class to determine the 3D TIN corresponding to the object pixels. The TTinView ( TTinView) is used to handle the viewing the output (see diagram schema in (Rahman 2008 pp. 137, 141)

\section{CONSIDERATIONS ON URBAN SPATIAL DATA APPLICATIONS}

Basing on the literature some considerations about these models can be made. A first group of issues involves the features of the objects we represent. The TEN model may be fit for the representation of irregular surfaces like archaeological layers which have irregular boundaries, the others are suitable for objects which have a regular shape and well defined boundaries (Zlatanova 2002). The other important aspects involving the 
suitability of the spatial models are those regarding the processing stages and the final target of the work, like the querying orientation or the visualization purposes. For example the increase of geometry subdivisions in the TEN and UDM models due to the tetrahedralization of bodies and triangulation of surfaces represent on the one hand a problem in the modelling stage, but it represents an advantage in the direction of the next stage: the visualisation. As underlined by Zlatanova (Zlatanova 2004) in the rendering step of the 3D visualisation every geometry is usually triangulated, and so this two models can be considered as two of the best for this purpose. Despite the tetrahedrons subdivision increases the database size, if we consider the visualisation purposes we notice that usually in a rendering pipeline culling procedures are adopted to determine the visible surface and so hidden geometry is usually discarded in the final computation.

Considering the explicit storage of the topological relationship we can say that a formal representation of topological relationships is useful especially in large dataset queries to reduce the amount of data retrieved in metric computations. For example if the topological relationship are embedded, i.e. stored together with the geometry informations, the adjacency query on the coordinates table is slower in comparison to a query performed in the topological relationships table. The topological query is faster due to the fact that is not necessary to perform distance analysis to identify the selected adjacent objects. Studies in this direction have been made by Oosterom (Oosterom 2002) involving the translation of the topological model in the geometrical one and the implementation in a ORDBMS (Object-Relational Management System). They were focused on balancing the advantage of data redundancy reduction, by mean of topological indexing, with the suitability of an explicit storage of geometrical primitives for querying, analysing and displaying purposes.

Regarding the structure of the data in the DBMS several studies stated the best attitude of the OODBMS to manage spatial data also proved by the implementation of $\mathrm{OO}$ spatial models from which the 3D TIN is an example. Egenhofer and Frank (Egenhofer, Franck 1992) investigated and formalised the most relevant aspects of this issue such as the ability to handle complex objects (like cities dataset), the possibility to do operations directly on them considered wholly and the capability to perform target queries on specific features which are stored in the embedded objects methods.

\section{OPEN ISSUES IN ARCHICTECTURAL HERITEGE SPATIAL DATA INTEGRATION}

As said before the multiscale approach plays a central role guiding the approach of a surveyor when he has to interfaces with architectural heritage. It rules the level of detail of the information to collect in a close relationship both with the cultural purposes of documentation and the needs of the information users. Some spatial models have been exposed in relation to the management of urban features and their geometrical and topological aspects have been summarized, so the question is "which role they can play in the architectural features modelling for architectural features for documentation and analysis purposes". As is quite agreed more than one spatial model is necessary to cover the needs of the applications categories involved in the urban management (Zlatanova 2004), but despite this there is a need of an integrated applications framework to manage different source of data, from the common architectonic $2 \mathrm{D}$ sections or $3 \mathrm{D}$ models to the LiDAR point clouds. In this field the 3D GIS software development is going in the direction of an integration of unstructured data such as point clouds or TINs like in the Spatial module of ORACLE Database11g which supports this kind of geometries (Kothuri 2007).

Considering the architectural thematic class of spatial data we can see that the number and complexity of the objects increases as far as the level of detail of the objects represented. In the LOD4 of CityGML Specification the representation scale is very close to the architectural scale. Many thematic classes like building furniture, building installation, window, doors and room classes are introduced. An important part of the specification is also directed to determines the exterior aspect of surfaces through many feature classes like materials and textures defined in the appearance module of the standard. This level of detail is very close to the one used in the AEC (Architectural, Engineering, Construction) sector to manage the flow of information through the steps of the building life from the planning to the maintenance management. Studies on the translation between thematic classes from the BIM approach in the building information management to GIS approach in the representation of urban features have been made (Zlatanova 2009). Other efforts on the application of CAD and GIS systems are also in the same direction towards the integration of the two fields of interests (Karimi 2010). Many of the studies involves the BIM or CAD to GIS translation because it is applied to the urban data management of building models coming from the AEC sector and so it's in the logical sequence of the data flow.

On the contrary the surveyed metric informations are usually organized and structured to be used by architects and engineers to build their projects of restoration. So in architectural heritage metric documentation generally the data flow is both from CAD to GIS and from GIS to CAD spatial data representations, maybe soon in the next years also from urban spatial models to BIM semantic models and viceversa. This involves a shift between the approach to architectural heritage spatial data management to the urban scale spatial data management. This involves differences in semantic data mapping and geometrical features representations.

The third-dimensional perspective on building spatial information management is assumed as a matter of fact whereas the 3D GIS is still an open issue for researches. On the other side the semantic mapping of building geometrical informations isn't yet a standard gained by the best practises. In this context the definition of spatial models in the GIS data structuring can assumes a central role if we consider that the geometry data are reused in the following stage of the architectural planning. A interoperability scheme for the geometrical data structuring from $3 \mathrm{D}$ GIS to BIM can be useful to avoid restructuring informations in the planning stage.

\section{REFERENCES}

Atkinson, M.; Bancilhon, F.; De Witt, D.; Dittrich, K.; Maier, D. \& Zdonik, S. (1990), The Object-Oriented Database System Manifesto, in H. V. Jagadish \& Hector Garcia-Molina, ed., Proceedings of the ACM SIGMOD International Conference on Management of Data, ACM Press, .

Carlson, E., (1987), Three dimensional conceptual modelling of subsurface structures, Technical Papers of ASPRS/ACSM Annual Convention, Baltimore, Vol. 4 (Cartography), pp. 188200 . 
Chiabrando F, Spano' A., 2008, Multiple Sensors and Multiresolution Data for Large Scale mapping and Artifacts Documentation, In: Advances in Remote Sensing for Archaeology and Cultural Heritage Management, Aracne editrice (ITA).

Codd, E. F. (1970), A Relational Model of Data for Large Shared Data Banks, Communication of the ACM XIII(6), 377387.

Coors, V., 2003. 3D GIS in Networking environments, Computer, Environment and Urban Systems, Vol. 27 (4), pp. 345-357.

Egenhofer, M. J. \& Franck, A. U. (1992), Object Oriented Modeling for GIS, URISA Journal IV(2), 3-19.

Karimi Hassan A, A. B., ed. (2010), CAD and GIS Integration, Taylor \& Francis.

Kothuri Ravi, Godfrind Albert, B. E. (2007), Pro Oracle Spatial for Oracle Database 11g, Apress.

Molenaar, M. (1990), A formal data structure for 3D vector maps, in: Proceedings of EGIS'90, Vol. 2, Amsterdam, The Netherlands, pp. 770-781

Molenaar, M. (1998), An Introduction to the Theory of Spatial Object Modelling for GIS, Taylor and Francis, London UK.

Oosterom, P. v.; Zlatanova, S.; Stoter, J. \& Quak, W. (2002), The Balance between Geometry and Topology, in Dianne Richardson \& Peter van Oosterom, ed., 'Advances in Spatial Data Handling', Springer-Verlag, , pp. 209-224.

Oosterom, P. v.; Jansen, E. \& Stoter, J.Zlatanova, S. \& Prosperi, D., ed., (2006), Large-scale 3D Data Integration: Challenges and Opportunities, Taylor and Francis - CRC Press, Boca Raton US-FL, chapter 1 Bridging the worlds of CAD and GIS, pp. 9-36.

Penninga, F. (2008), 3D Topography, PhD thesis, Delft University of Technology.

Pigot, S. (1991), Topological Models for 3D Spatial Information Systems, in 'Proceedings of the International Symposium on Computer-Assisted Cartography', pp. 368-391. Pilouk, M. (1996), Integrated modelling for 3D GIS', PhD thesis, ITC - University of Twente.

Rahman, A., 2000, The design and implementation of two and three-dimensional triangular irregular network (TIN) based GIS, PhD thesis, University of Glasgow, United Kingdom.
Rahman, A. A. \& Pilouk, M. (2008), Spatial Data Modelling for $3 D$ GIS, Springer-Verlag, Berlin Heidelberg.

Spano' A., 2008, La cartografia di Hierapolis, Archiviazione in database topografico e rappresentazione di dati archeologici e territoriali, In: Atlante di Hierapolis di Frigia, F. Dandria; G. Scardozzi; A. Spano' (ed), Ege Yayinlari (TUR), 2008, pp. 1-9. Spano' A., Bonfanti C., 2005, Large scale spatial database supporting archaeological research, ISPRS Archives, Vol. VOL. XXXVI, pp 963-968.

Spano' A., M. Pellegrino, 2010, Multi-content architectural archives for complex framework testing. A case study., In: "Science and Technology for the Safeguard of Cultural Heritage of the Mediterranean Basin", Cairo 6-8 dicembre 2009,2010

Zlatanova, S. (2000), 3D GIS for Urban Development, PhD thesis, Graz University of Technology.

Zlatanova, S.; Rahman, A. A. \& Pilouk, M. (2002), 3D GIS: Current Status and Perspectives, in The International Archives of the Photogrammetry, Remote Sensing and Spatial Information Sciences.

Zlatanova, S.; Rahman, A. A. \& Shi, W. (2004), Topological models and frameworks for 3D spatial objects, Journal of Computers and Geosciences XXX(4), 419-428.

Zlatanova, S. \& Isikdag, U.Zlatanova, S. \& Jiyeong, L., ed., (2009), Towards Defining a Framework for Automatic Generation of Buildings in CityGML Using BIM, SpringerVerlag, Berlin Heidelberg DE, pp. 79-96.

CityGML, OpenGIS ${ }^{\circledR}$ City Geography Markup Language Encoding Standard (v. 3.1.1 GML3). 